\title{
MIXED AUTOMORPHIC FORMS ON SEMISIMPLE LIE GROUPS
}

\author{
MIN Ho LEE
}

\section{Introduction}

Let $\Gamma$ be a discrete subgroup of $\operatorname{PSL}(2, \mathbb{R})$ and let $\chi: \Gamma \rightarrow S L(2, \mathbb{R})$ be a homomorphism of groups. Then both $\Gamma$ and $\chi(\Gamma)$ operate on the Poincaré upper half plane $\mathcal{H}$ by linear fractional transformations. We assume that there is a holomorphic map $\omega: \mathcal{H} \rightarrow \mathcal{H}$ satisfying $\omega(g z)=\chi(g) \omega(z)$ for all $g \in \Gamma$ and $z \in \mathcal{H}$. Given a pair of nonnegative integers $k$ and $l$ with $k$ even, a holomorphic function $f: \mathcal{H} \rightarrow \mathbb{C}$ satisfying the condition

$$
f(g z)=(c z+d)^{k}\left(c_{\chi} \omega(z)+d_{\chi}\right)^{l} f(z)
$$

for all $z \in \mathcal{H}$ and

$$
g=\left(\begin{array}{ll}
a & b \\
c & d
\end{array}\right) \in \Gamma, \quad \chi(g)=\left(\begin{array}{ll}
a_{\chi} & b_{\chi} \\
c_{\chi} & d_{\chi}
\end{array}\right) \in S L(2, \mathbb{R})
$$

is a holomorphic mixed automorphic form of one variable of type $(k, l)$ associated to $\Gamma$, $\omega$ and $\chi$ if $f$ satisfies an additional condition of regularity at the cusps of $\Gamma$ (see [16]). Certain types of such mixed automorphic forms occur naturally as holomorphic forms of the highest degree on elliptic varieties which are fiber varieties over an arithmetic variety with generic fiber a product of elliptic curves (cf. [10], [15]). Holomorphic mixed automorphic forms of several variables were also introduced in [17] and [18], and it was proved that a certain class of such automorphic forms can be interpreted as holomorphic forms on some families of abelian varieties over an arithmetic variety.

The purpose of this paper is to describe mixed automorphic forms in the setting of representations of semisimple Lie groups following such descriptions for the usual automorphic forms initiated by Selberg and Langlands (e.g., see [3], [4], [9], [21]). More specifically, we define mixed automorphic forms on semisimple Lie groups which generalize holomorphic mixed automorphic forms, and construct Poincaré series and Eisenstein series for such automorphic forms.

\section{Mixed automorphic forms on a semisimple Lie group}

First, we shall review the definition of the usual automorphic forms on semisimple Lie groups (e.g., see [3], [4], [5], [6]). Let $G$ be a semisimple Lie group, and let $\mathfrak{g}$ be

Received May 5, 1995.

1991 Mathematics Subject Classification. Primary 11F55; Seconadary 11F70, 22E46. 
its Lie algebra. If $V$ is a finite-dimensional complex vector space, then $\mathfrak{g}$ operates on smooth functions $f: G \rightarrow V$ by

$$
(Y \because f)(g)=\left.\frac{d}{d t} f((\exp t Y) g)\right|_{t=0}
$$

for $Y \in \mathfrak{g}$ and $g \in G$. Let $Z(\mathfrak{g})$ be the center of the universal enveloping algebra $U(\mathfrak{g})$ of the complexification $\mathfrak{g}_{\mathbb{C}}$ of $\mathfrak{g}$. Then a vector-valued function $f: G \rightarrow V$ is said to be $Z(\mathfrak{g})$-finite if $Z(\mathfrak{g}) \cdot f$ is a finite-dimensional vector space. If $\alpha: G \rightarrow G L(W)$ is a finite-dimensional complex representation whose kernel is finite and whose image is closed in $\operatorname{End}(W)$, then we can define a norm $\|\cdot\|_{\alpha}$ on $G$ by

$$
\|g\|_{\alpha}=\left(\operatorname{Tr}\left(\alpha(g)^{*} \cdot \alpha(g)\right)\right)^{1 / 2}
$$

where $*$ denotes the adjoint with respect to a Hilbert space structure on $W$ invariant under a maximal compact subgroup $K$ of $G$. If $\beta$ is another such representation, then there is a constant $M>0$ and a positive integer $m$ such that

$$
\|x\|_{\alpha} \leq M\|x\|_{\beta}^{m}
$$

for all $x \in G$. A vector valued function $f: G \rightarrow V$ is said to be slowly increasing if there is a norm $\|\cdot\|$ on $G$, a constant $C>0$, and a positive integer $m$ such that

$$
|f(g)| \leq C\|g\|^{m}
$$

where $|\cdot|$ is a norm on $V$.

Definition 2.1. Let $K$ be a maximal compact subgroup of $G, \Gamma$ a discrete subgroup of $G$, and $\sigma: K \rightarrow G L(V)$ a representation of $K$ in a finite-dimensional complex vector space $V$. A smooth vector-valued function $f: G \rightarrow V$ is an automorphic form for $\Gamma$ and $\sigma$ if the following conditions are satisfied:

(i) $f(k g \gamma)=\sigma(k) f(g)$ for all $k \in K$ and $\gamma \in \Gamma$.

(ii) $f$ is $Z(\mathfrak{g})$-finite.

(iii) $f$ is slowly increasing.

Let $G, K, \Gamma$, and the representation $\sigma: K \rightarrow G L(V)$ be as in Definition 2.1, and consider another semisimple Lie group $G^{\prime}$. Let $K^{\prime}$ be a maximal compact subgroup and $\Gamma^{\prime}$ a discrete subgroup of $G^{\prime}$. Let $\rho: G \rightarrow G^{\prime}$ be a homomorphism such that $\rho(K) \subset K^{\prime}$ and $\rho(\Gamma) \subset \Gamma^{\prime}$, and let $\sigma^{\prime}: K^{\prime} \rightarrow G L\left(V^{\prime}\right)$ be a representation of $K^{\prime}$ in a finite-dimensional complex vector space $V^{\prime}$. Then we obtain the representation $\sigma \otimes\left(\left.\sigma^{\prime} \circ \rho\right|_{K}\right): K \rightarrow G L\left(V \otimes V^{\prime}\right)$ of $K$ in $V \otimes V^{\prime}$, where $\left.\rho\right|_{K}$ denotes the restriction of $\rho$ to $K$. 
Definition 2.2. A mixed automorphic form for $\Gamma$ of type $\left(\rho, \sigma, \sigma^{\prime}\right)$ is an automorphic form for $\Gamma$ and the representation $\sigma \otimes\left(\left.\sigma^{\prime} \circ \rho\right|_{K}\right)$.

Let $f_{\sigma}: G \rightarrow V$ (resp. $f_{\sigma^{\prime}}^{\prime}: G^{\prime} \rightarrow V^{\prime}$ ) be an automorphic form for $\Gamma$ (resp. $\Gamma^{\prime}$ ) and $\sigma: K \rightarrow G L(V)$ (resp. $\sigma^{\prime}: K^{\prime} \rightarrow G L\left(V^{\prime}\right)$ ), where $V$ (resp. $V^{\prime}$ ) is a finitedimensional complex vector space. We denote by $f_{\rho, \sigma, \sigma^{\prime}}$ the function from $G$ to $V \otimes V^{\prime}$ given by

$$
f_{\rho, \sigma, \sigma^{\prime}}(g)=\left(f_{\sigma} \otimes\left(f_{\sigma^{\prime}}^{\prime} \circ \rho\right)\right)(g)=f_{\sigma}(g) \otimes f_{\sigma^{\prime}}^{\prime}(\rho(g))
$$

for all $g \in G$.

PROPOSITION 2.3. The function $f_{\rho, \sigma, \sigma^{\prime}}: G \rightarrow V \otimes V^{\prime}$ described above is a mixed automorphic form for $\Gamma$ of type $\left(\rho, \sigma, \sigma^{\prime}\right)$.

Proof. We must show that $f_{\rho, \sigma, \sigma^{\prime}}$ satisfies the conditions (i), (ii) and (iii) in Definition 2.1 for the discrete group $\Gamma$ and the representation $\sigma \otimes\left(\left.\sigma^{\prime} \circ \rho\right|_{K}\right)$. For $g \in G, k \in K$ and $\gamma \in \Gamma$, we have

$$
\begin{aligned}
f_{\rho, \sigma, \sigma^{\prime}}(k g \gamma) & =f(k g \gamma) \otimes f^{\prime}(\rho(k) \rho(g) \rho(\gamma)) \\
& =\sigma(k) f(g) \otimes \sigma^{\prime}(\rho(k)) f^{\prime}(\rho(g)) \\
& =\left(\sigma \otimes\left(\left.\sigma^{\prime} \circ \rho\right|_{K}\right)\right)(k) \cdot\left(f \otimes f^{\prime} \circ \rho\right)(g),
\end{aligned}
$$

which shows the condition (i). Now, given $Y \in Z(\mathfrak{g})$, we have

$$
\begin{aligned}
Y \cdot f_{\rho, \sigma, \sigma^{\prime}}(g)= & \left.\frac{d}{d t} f_{\rho, \sigma, \sigma^{\prime}}((\exp t Y) g)\right|_{t=0} \\
= & {\left[\frac{d}{d t} f_{\sigma}((\exp t Y) g)\right]_{t=0} \otimes f_{\sigma^{\prime}}^{\prime}(\rho(g)) } \\
& +f_{\sigma}(g) \otimes\left[\frac{d}{d t} f_{\sigma^{\prime}}^{\prime}(\rho((\exp t Y) g))\right]_{t=0} \\
= & {\left[\frac{d}{d t} f_{\sigma}((\exp t Y) g)\right]_{t=0} \otimes f_{\sigma^{\prime}}^{\prime}(\rho(g)) } \\
& +f_{\sigma}(g) \otimes\left[\frac{d}{d t} f_{\sigma^{\prime}}^{\prime}((\exp t d \rho Y) \rho(g))\right]_{t=0} .
\end{aligned}
$$

Thus the condition (ii) follows from the fact that $f_{\sigma}$ is $Z(\mathfrak{g})$-finite and $f_{\sigma^{\prime}}^{\prime}$ is $Z\left(\mathfrak{g}^{\prime}\right)$ finite. As for the condition (iii), since $f_{\sigma}$ and $f_{\sigma^{\prime}}^{\prime}$ are slowly increasing, we have

$$
\left|f_{\rho, \sigma, \sigma^{\prime}}(g)\right|=\left|f_{\sigma}(g) \otimes f_{\sigma^{\prime}}^{\prime}(\rho(g))\right| \leq C_{1}\|g\|_{\alpha}^{m_{1}} \cdot C_{2}\|\rho(g)\|_{\beta}^{m_{2}}
$$


for some constants $C_{1}, C_{2}$, positive integers $m_{1}, m_{2}$, and representations $\alpha: G \rightarrow$ $G L(W), \beta: G \rightarrow G L\left(W^{\prime}\right)$. However, we have

$$
\|\rho(g)\|_{\beta}=\|g\|_{\beta \circ \rho} \leq C_{3}\|g\|_{\alpha}^{m_{3}}
$$

for some constant $C_{3}$ and positive integer $m_{3}$. Thus we have

$$
\left|f_{\rho, \sigma, \sigma^{\prime}}(g)\right| \leq C_{1} C_{2} C_{3}\|g\|_{\alpha}^{m_{1}+m_{3}},
$$

and $f_{\rho, \sigma, \sigma^{\prime}}$ is slowly increasing.

Example 2.4. Let $\rho: G \rightarrow G^{\prime}, K, K^{\prime}, V, V^{\prime}$ be as above. Assume that the symmetric spaces $D=G / K$ and $D^{\prime}=G^{\prime} / K^{\prime}$ have $G$-invariant and $G^{\prime}$-invariant complex structures, respectively. This assumption implies that $D$ and $D^{\prime}$ are equivalent to bounded symmetric domains (e.g., see [8]). Let $J: G \times D \rightarrow G L(V)$ and $J^{\prime}: G^{\prime} \times D^{\prime} \rightarrow G L\left(V^{\prime}\right)$ be automorphy factors, and let $\tau: D \rightarrow D^{\prime}$ be a holomorphic map satisfying

$$
\tau(g z)=\rho(g) \tau(z)
$$

for all $g \in G$ and $z \in D$. Then we can define a mixed automorphic vector bundle $\mathcal{M}$ (see [18]) on the Shimura variety $X=\Gamma \backslash D$ whose sections can be considered as holomorphic mappings $f: D \rightarrow V \otimes V^{\prime}$ satisfying

$$
f(g z)=\left(J(g, z) \otimes J^{\prime}(\rho(g), \tau(z))\right) f(z)
$$

for all $g \in G$ and $z \in D$. Given such a bundle $\mathcal{M}$ associated to $J, J^{\prime}, \rho$ and $\tau$, we define mappings $\sigma: G \rightarrow G L(V)$ and $\sigma^{\prime}: G^{\prime} \rightarrow G L\left(V^{\prime}\right)$ by

$$
\sigma(k)=J(k, 0), \quad \sigma^{\prime}\left(k^{\prime}\right)=J^{\prime}\left(k^{\prime}, 0^{\prime}\right),
$$

where $0 \in D$ and $0^{\prime} \in D^{\prime}$ are the fixed points of $K$ and $K^{\prime}$, respectively. Then $\sigma$ and $\sigma^{\prime}$ are representations of $G$ and $G^{\prime}$, respectively, and the sections of $\mathcal{M}$ are mixed automorphic forms for $\Gamma$ of type $\left(\rho, \sigma, \sigma^{\prime}\right)$.

Remark 2.5. In Example 2.4, if $G^{\prime}$ is a symplectic group and if $\Gamma$ is cocompact, then it was shown in [18] that for some specific automorphy factors $J$ and $J^{\prime}$ the sections of the mixed automorphic vector bundle $\mathcal{M}$ can be identified with holomorphic forms on certain families of abelian varieties parametrized by a Shimura variety. In the elliptic modular case, that is, when $G=S L(2, \mathbb{R})$, various results have been obtained concerning the corresponding mixed automorphic forms (e.g., see [10], [15], [16]). Similar geometric aspects for the Siegel modular case were treated in [17]. 


\section{Poincaré series}

In this section, as our first examples of mixed automorphic forms, we shall construct Poincaré series. Let $\Gamma$ (resp. $\Gamma^{\prime}$ ) be a discrete subgroup of a semisimple Lie group $G$ (resp. $G^{\prime}$ ), and let $K$ (resp. $K^{\prime}$ ) be a maximal compact subgroup of $G$ (resp. $G^{\prime}$ ). Let $\rho: G \rightarrow G^{\prime}$ be a homomorphism such that $\rho(K) \subset K^{\prime}$. If $f: G \rightarrow V$ is a vector-valued function and if $h$ is an element of $G$, we denote by $l(h)$ and $r(h)$ the translation operators given by

$$
l(h) f(g)=f\left(h^{-1} g\right), \quad r(h) f(g)=f(g h)
$$

for all $g \in G$.

Definition 3.1. A vector-valued function $f: G \rightarrow V$ on $G$ is said to be left (resp. right) $K$-finite if the set of left (resp. right) translations

$$
\{l(k) f \mid k \in K\} \quad(\text { resp. }\{r(k) f \mid k \in K\})
$$

of $f$ by elements of $K$ spans a finite-dimensional vector space.

PROPOSITION 3.2. Let $V$ and $V^{\prime}$ be finite-dimensional complex vector spaces, and assume that the following conditions are satisfied:

(i) $f \otimes\left(f^{\prime} \circ \rho\right) \in L^{1}(G) \otimes\left(V \otimes V^{\prime}\right)$, where $L^{1}(G)$ denotes the set of integrable functions on $G$.

(ii) $f$ is $Z(\mathfrak{g})$-finite and $f^{\prime}$ is $Z\left(\mathfrak{g}^{\prime}\right)$-finite.

(iii) $f$ is right $K$-finite and $f^{\prime}$ is right $K^{\prime}$-finite.

Then the series $P_{\rho, f, f^{\prime}}(g)$ defined by

$$
P_{\rho, f, f^{\prime}}(g)=\sum_{\gamma \in \Gamma}\left(f \otimes\left(f^{\prime} \circ \rho\right)\right)(g \cdot \gamma)
$$

converges absolutely and uniformly on compact sets. Furthermore, the series

$$
\sum_{\gamma \in \Gamma}\left|\left(f \otimes\left(f^{\prime} \circ \rho\right)\right)(g \cdot \gamma)\right|
$$

is bounded on $G$ where $|\cdot|$ denotes the norm on $V \otimes V^{\prime}$.

Proof. As in the proof of Proposition 2.3 we can show that the function $f \otimes\left(f^{\prime} \circ\right.$ $\rho$ ): $G \rightarrow V \otimes V^{\prime}$ is $Z$ (g)-finite using condition (ii). For $k \in K$ and $g \in G$, we have

$$
\begin{aligned}
r(k)\left(f \otimes\left(f^{\prime} \circ \rho\right)\right)(g) & =\left(f \otimes\left(f^{\prime} \circ \rho\right)\right) \\
(g k) & =f(g k) \otimes f^{\prime}(\rho(g) \rho(k)) \\
& =r(k) f(g) \otimes r(\rho(k)) f^{\prime}(\rho(k)) .
\end{aligned}
$$


Hence condition (iii) implies that $f \otimes\left(f^{\prime} \circ \rho\right)$ is right $K$-finite. Therefore the proposition follows from [1, Theorem 23], [2, Theorem 5.4], or [3, Theorem 9.1].

Definition 3.3. The series

$$
P_{\rho, f, f^{\prime}}(g)=\sum_{\gamma \in \Gamma}\left(f \otimes\left(f^{\prime} \circ \rho\right)\right)(g \cdot \gamma)
$$

is called a Poincaré series associated to $\rho, f$ and $f^{\prime}$.

COROLlary 3.4. Let $\sigma: K \rightarrow G L(V)$ and $\sigma^{\prime}: K^{\prime} \rightarrow G L\left(V^{\prime}\right)$ be finite-dimensional representations of $K$ and $K^{\prime}$, respectively. Assume the $f$ and $f^{\prime}$ satisfy the conditions (i), (ii) and (iii) of Proposition 3.2 together with the condition that

$$
f(k g)=\sigma(k) f(g), \quad f^{\prime}\left(k^{\prime} g^{\prime}\right)=\sigma^{\prime}\left(k^{\prime}\right) f^{\prime}\left(g^{\prime}\right)
$$

for $k \in K, g \in G, k^{\prime} \in K^{\prime}$ and $g^{\prime} \in G^{\prime}$. Then the Poincaré series $P_{\rho, f, f^{\prime}}$ is a mixed automorphic form for $\Gamma$ of type $\left(\rho, \sigma, \sigma^{\prime}\right)$.

Proof. Since $f \otimes\left(f^{\prime} \circ \rho\right)$ is $Z(\mathfrak{g})$-finite on the left, so is the Poincare series $P_{\rho, f, f^{\prime}}$. From the definition of $P_{\rho, f, f^{\prime}}(g)$ the right $\Gamma$-invariance of $P_{\rho, f, f^{\prime}}$ follows immediately, and $P_{\rho, f, f^{\prime}}$ is slowly increasing by Proposition 3.2. As in the proof of Proposition 2.3, we have

$$
\left(f \otimes\left(f^{\prime} \circ \rho\right)\right)(k g)=\left(\sigma \otimes\left(\left.\sigma^{\prime} \circ \rho\right|_{K}\right)\right)(k)\left(f \otimes\left(f^{\prime} \circ \rho\right)\right)(g) ;
$$

hence it follows that

$$
P_{\rho, f, f^{\prime}}(k g)=\left(\sigma \otimes\left(\left.\sigma^{\prime} \circ \rho\right|_{K}\right)\right)(k) P_{\rho, f, f^{\prime}}(g),
$$

and the proof of the corollary is complete.

Let $G, G^{\prime}, K, K^{\prime}, \rho$, and $\tau$ be as in Example 2.4. In particular, $D=G / K$ and $D^{\prime}=G^{\prime} / K^{\prime}$ are symmetric domains, and $\tau: D \rightarrow D^{\prime}$ is a holomorphic mapping satisfying

$$
\tau(g z)=\rho(g) \tau(z)
$$

for all $g \in G$ and $z \in D$. Let $J: G \times D \rightarrow G L(V)$ (resp. $J^{\prime}: G^{\prime} \times D^{\prime} \rightarrow G L\left(V^{\prime}\right)$ ) be the canonical automorphy factor (see [2, §1.8], [20, §II.5]), and let $j: G \times D \rightarrow \mathbb{C}$ (resp. $j^{\prime}: G^{\prime} \times D^{\prime} \rightarrow \mathbb{C}$ ) be the corresponding Jacobian determinant function.

LEMMA 3.5. If $l$ and $m$ are nonnegative integers with $l \geq 2$, then the mapping

$$
g \mapsto j(g, 0)^{l} \otimes j^{\prime}\left(\rho(g), 0^{\prime}\right)^{m}: G \rightarrow \mathbb{C}
$$

is in $L^{1}(G)$. 
Proof. The mapping $g \mapsto|j(g, 0)|^{l}$ (resp. $\left.g^{\prime} \mapsto\left|j^{\prime}\left(g^{\prime}, 0^{\prime}\right)\right|^{m}\right)$ is left and right $K$ invariant (resp. $K^{\prime}$-invariant) (see $\left.[2, \S 5.8]\right)$; hence it can be considered as a function on $D$ (resp. $\left.D^{\prime}\right)$, and we have

$$
\int_{G}\left|j(g, 0)^{l} j^{\prime}\left(\rho(g), 0^{\prime}\right)^{m}\right| d g=\int_{D}|j(z, 0)|^{l}\left|j^{\prime}\left(\tau(z), 0^{\prime}\right)\right|^{m} d \mu(z),
$$

where $d \mu(z)$ denotes the invariant Bergmann measure on $D$ (cf. [1, §4.3]). If $d z$ is the usual Euclidean measure on $D$, then we have

$$
d \mu(z)=|j(z, 0)|^{-2} d z
$$

up to a positive factor. Thus we have

$$
\int_{G}\left|j(g, 0)^{l} j^{\prime}\left(\rho(g), 0^{\prime}\right)^{m}\right| d g=\int_{D}|j(z, 0)|^{l-2}\left|j^{\prime}\left(\tau(z), 0^{\prime}\right)\right|^{m} d z .
$$

However, both $|j(z, 0)|$ and $\left|j\left(\tau(z), 0^{\prime}\right)\right|$ are bounded by [2, Proposition 1.12]; hence the lemma follows.

Let $F: D \rightarrow V$ and $F^{\prime}: D^{\prime} \rightarrow V^{\prime}$ be mappings such that $F \otimes\left(F^{\prime} \circ \tau\right): D \rightarrow V \otimes V^{\prime}$ is a polynomial mapping. Given nonnegative integers $l, m$ with $l \geq 2$, we define the functions $f: G \rightarrow V$ and $f^{\prime}: G^{\prime} \rightarrow V^{\prime}$ by

$$
f(g)=j(g, 0)^{l} F(\pi g), \quad f^{\prime}\left(g^{\prime}\right)=j^{\prime}\left(g^{\prime}, 0^{\prime}\right)^{m} F^{\prime}\left(\pi^{\prime} g^{\prime}\right)
$$

for $g \in G$ and $g^{\prime} \in G^{\prime}$, where $0 \in D$ and $0^{\prime} \in D^{\prime}$ are the fixed points of $K$ and $K^{\prime}$, respectively, and $\pi: G \rightarrow D$ and $\pi^{\prime}: G^{\prime} \rightarrow D^{\prime}$ are canonical projection mappings. We set

$$
\sigma(k)=j(k, 0)^{l}, \quad \sigma^{\prime}\left(k^{\prime}\right)=j\left(k^{\prime}, 0^{\prime}\right)^{m}
$$

for $k \in K$ and $k^{\prime} \in K^{\prime}$. Then $\sigma$ and $\sigma^{\prime}$ are representations of $G$ and $G^{\prime}$ in $V$ and $V^{\prime}$, respectively. We set

$$
P_{\rho, j, j^{\prime}}^{l, m}(g)=\sum_{\gamma \in \Gamma}\left(f \otimes\left(f^{\prime} \circ \rho\right)\right)(g \gamma)
$$

for $g \in G$, which is the Poincare series $P_{\rho, f, f^{\prime}}$ in the sense of Definition 3.3.

THEOREM 3.6. The Poincaré series $P_{\rho, j, j^{\prime}}^{l, m}(g)$ is a mixed automorphic form for $\Gamma$ of type $\left(\rho, \sigma, \sigma^{\prime}\right)$.

Proof. For each $g \in G$ we have

$$
\begin{aligned}
\left(f \otimes\left(f^{\prime} \circ \rho\right)\right)(g) & =\left(j(g, 0)^{l} F(\pi g)\right) \otimes\left(j^{\prime}\left(\rho(g), 0^{\prime}\right)^{m} F^{\prime}\left(\pi^{\prime}(\rho(g))\right)\right) \\
& =\left(j(g, 0)^{l} \cdot j^{\prime}\left(\rho(g), 0^{\prime}\right)^{m}\right)\left(F(\pi g) \otimes F^{\prime}(\omega(\pi g))\right) .
\end{aligned}
$$


Thus from Lemma 3.5 and the fact that $F \otimes\left(F^{\prime} \circ \omega\right)$ is a polynomial mapping on the bounded symmetric domain $D$ it follows that

$$
\int_{G}\left|\left(f \otimes\left(f^{\prime} \circ \rho\right)\right)(g)\right| d g<\infty ;
$$

hence $f \otimes\left(f^{\prime} \circ \rho\right)$ is in $L^{1}(G) \otimes\left(V \otimes V^{\prime}\right)$. Since $f(k g)=\sigma(k) f(g)$ and $f^{\prime}\left(k^{\prime} g^{\prime}\right)=$ $\sigma^{\prime}\left(k^{\prime}\right) f^{\prime}\left(g^{\prime}\right)$ for $k \in K, k^{\prime} \in K^{\prime}, g \in G$ and $g^{\prime} \in G^{\prime}$, it follows that $f$ is $Z_{\mathfrak{g}}$-finite and $f^{\prime}$ is $Z_{\mathfrak{g}^{\prime}}$-finite. For $k \in K$ and $g \in G$ we have $j(g k, 0)=j(g, 0) j(k, 0)$, and the set of right translates $r(k) F(\pi(g k))$ are polynomials of the same degree as $F$; hence $f$ is right $K$ - finite. Similarly, $f^{\prime}$ is right $K^{\prime}$-finite. Thus it follows from Proposition 3.2 that the series $\sum_{\gamma \in \Gamma}\left|\left(f \otimes\left(f^{\prime} \circ \rho\right)\right)(g \gamma)\right|$ is bounded on $G$. In particular, $P_{\rho, j, j^{\prime}}^{l, m}$ is slowly increasing. As in the proof of Proposition 2.3, $f \otimes\left(f^{\prime} \circ \rho\right)$ is $Z_{\mathfrak{g}}$-finite, and for $k \in K$ and $g \in G$ we have

$$
\left(f \otimes\left(f^{\prime} \circ \rho\right)\right)(k g)=\left(\sigma \otimes\left(\left.\sigma^{\prime} \circ \rho\right|_{K}\right)\right)(k)\left(f \otimes\left(f^{\prime} \circ \rho\right)\right)(g)
$$

hence it follows that

$$
P_{\rho, j, j^{\prime}}^{l, m}(k g)=\left(\sigma \otimes\left(\left.\sigma^{\prime} \circ \rho\right|_{K}\right)\right)(k) P_{\rho, j, j^{\prime}}^{l, m}(g)
$$

Therefore $P_{\rho, j, j^{\prime}}^{l, m}(g)$ is an automorphic form for $\Gamma$ of type $\left(\rho, \sigma, \sigma^{\prime}\right)$ in the sense of Definition 2.1.

\section{Eisenstein series}

In order to discuss Eisenstein series, instead of the usual semisimple Lie group $G$ we shall first consider an algebraic group whose set of real points will coincide with $G$. Let $\mathbb{G}$ be a connected, semisimple, linear algebraic group defined over a subfield $k$ of $\mathbb{R}$, and let $\mathbb{P}$ be a $k$-parabolic subgroup of $\mathbb{G}$ containing a maximal $k$-split torus $\mathbb{S}$ of $\mathbb{G}$. Let $\mathbb{P}_{0}$ be a minimal $k$-parabolic subgroup of $\mathbb{G}$ such that $\mathbb{S} \subset \mathbb{P}_{0} \subset \mathbb{P}$. We define an ordering on the set $\Sigma_{k}$ of $k$-roots of $\mathbb{G}$ with respect to $\mathbb{S}$ as follows: $\mathrm{A} k$-root $\alpha \in \Sigma_{k}$ is positive if and only if the subgroup of $\mathbb{G}$ generated by the $\alpha$-eigenspace of the adjoint representation of $\mathbb{S}$ is contained in $\mathbb{P}_{0}$. We denote by $\Delta_{k}$ the set of simple positive $k$-roots in $\Sigma_{k}$.

If $\Xi$ is a subset of $\Delta_{k}$, we set

$$
\mathbb{S}_{\Xi}=\left(\bigcap_{\alpha \in \Xi} \operatorname{Ker} \alpha\right)^{0}
$$

where ( $)^{0}$ denotes the connected component of the identity. Let $\Theta$ be the subset of $\Delta_{k}$ such that $\mathbb{P}$ is generated by the unipotent radical $\mathbb{U}_{0}$ of $\mathbb{P}_{0}$ and by the centralizer 
$Z\left(\mathbb{S}_{\Theta}\right)$ of $\mathbb{S}_{\Theta}$. Let $\mathbb{U}_{\Theta}$ be the unipotent radical of $\mathbb{P}$, and let $\mathbb{M}_{\Theta}$ be a subgroup of $\mathbb{G}$ such that $Z_{\mathbb{G}}\left(\mathbb{S}_{\Theta}\right)=\mathbb{S}_{\Theta} \cdot \mathbb{M}_{\Theta}$ with $\mathbb{S}_{\Theta} \cap \mathbb{M}_{\Theta}$ finite. We set

$$
P=\mathbb{P}(\mathbb{R}), \quad A=\mathbb{S}_{\Theta}(\mathbb{R})^{0}, \quad M=\mathbb{M}_{\Theta}(\mathbb{R}), \quad U=\mathbb{U}_{\Theta}(\mathbb{R}) .
$$

Then we obtain the Langlands decomposition

$$
P=M A U
$$

of $P$ and the corresponding decomposition

$$
G=K P=K M A U
$$

of $G=G(\mathbb{R})$. If $g=k m a u \in G$ with $k \in K, m \in M, a \in A$ and $u \in U$, then $k \cdot m$, $a$ and $u$ are uniquely determined. We write $a=a(g)$.

Let $\left\{\Lambda_{\alpha}\right\}_{\alpha \in \Delta_{k}}$ be the set of fundamental dominant $k$-weights of $\mathbb{G}$ that satisfy

$$
\left\langle\Lambda_{\alpha}, \beta\right\rangle=d_{\alpha} \delta_{\alpha \beta}
$$

for all $\alpha, \beta \in \Delta_{k}$, where $\delta_{\alpha \beta}$ is the Kronecker delta and $d_{\alpha}$ is a positive real number (see $[3, \S 11]$ ). Let $\mathfrak{u}$ be the Lie algebra of $U$ and let $\chi=\operatorname{det} \operatorname{Ad}_{\mathfrak{u}}$ be the character of $P$ with $p^{\chi}=\operatorname{det} \operatorname{Ad}_{\mathfrak{u}} p$ for $p \in P$. We set $\tilde{\Theta}=\Delta_{k}-\Theta$. Then $\chi$ is a positive linear combination of the $\Lambda_{\alpha}$ for $\alpha \in \tilde{\Theta}$, i.e.,

$$
\chi=\sum_{\alpha \in \tilde{\Theta}} e_{\alpha} \Lambda_{\alpha} \quad \text { with } e_{\alpha}>0 .
$$

If $\left\{s_{\alpha}\right\}_{\alpha \in \tilde{\Theta}}$ is a set of complex numbers $s_{\alpha} \in \mathbb{C}$ associated to each $\alpha \in \tilde{\Theta}$, and if $p \in P$, then we set

$$
p^{\Lambda_{s}}=\prod_{\alpha \in \tilde{\Theta}}\left|p^{\Lambda_{\alpha}}\right|^{s_{\alpha}} .
$$

LEMMA 4.1. Let $\Gamma$ be a discrete subgroup of $G$ and let $\Gamma_{\infty}$ be a subgroup of $\Gamma \cap M U$. Suppose that there is a set $\left\{s_{\alpha}\right\}_{\alpha \in \tilde{\Theta}}$ of complex numbers satisfying the following conditions:

(i) $a(\gamma)^{\Lambda_{\alpha}} \geq \epsilon>0$ for all $\gamma \in \Gamma$ and $\alpha \in \tilde{\Theta}$.

(ii) $M U / \Gamma_{\infty}$ has finite measure.

(iii) $\operatorname{Re} s_{\alpha}>e_{\alpha}$ for all $\alpha \in \tilde{\Theta}$.

Then the series

$$
E(g, s)=\sum_{\gamma \in \Gamma / \Gamma_{\infty}} a(g \gamma)^{-\Lambda_{s}}
$$

converges uniformly on any compact subset of $G$. 
Proof. See Lemma 4 in [1] or Lemma 11.1 in [3] (see also [7]).

Let $G^{\prime}=\mathbb{G}^{\prime}(\mathbb{R})$ be another semisimple Lie group associated to a connected algebraic group $\mathbb{G}^{\prime}$ defined over a subfield $k$ of $\mathbb{R}$. We consider the corresponding subgroups $K^{\prime}, P^{\prime}, M^{\prime}, A^{\prime}, U^{\prime}$, etc. defined in a way similar to the case of $G$ above. Thus we have decompositions

$$
P^{\prime}=K^{\prime} A^{\prime} U^{\prime}, \quad G^{\prime}=K^{\prime} P^{\prime}=K^{\prime} M^{\prime} A^{\prime} U^{\prime} .
$$

Let $\rho: G \rightarrow G^{\prime}$ be a Lie group homomorphism such that

$$
\rho(K) \subset K^{\prime}, \quad \rho(P) \subset P^{\prime}, \quad \rho(A) \subset A^{\prime} .
$$

THEOREM 4.2. Let $\Gamma$ and $\Gamma_{\infty}$ be as in Lemma 4.1, and let $f: G \rightarrow V$ and $f^{\prime}: G^{\prime} \rightarrow V^{\prime}$ be smooth vector-valued functions, where $V$ and $V^{\prime}$ are finite-dimensional vector spaces. Suppose that there is a set $\left\{s_{\alpha}\right\}_{\alpha \in \tilde{\Theta}}$ of complex numbers satisfying the following conditions:

(i) $a(\gamma)^{\Lambda_{\alpha}} \geq \epsilon>0$ for all $\gamma \in \Gamma$ and $\alpha \in \tilde{\Theta}$.

(ii) $M U / \Gamma_{\infty}$ has finite measure.

(iii) $\operatorname{Re} s_{\alpha}>e_{\alpha}$ for all $\alpha \in \tilde{\Theta}$.

(iv) $\left(f \otimes\left(f^{\prime} \circ \rho\right)\right)(g \gamma)=\left(f \otimes\left(f^{\prime} \circ \rho\right)\right)(g)$ for all $\gamma \in \Gamma_{\infty}$.

(v) $|f(g p)|\left|f^{\prime}(\rho(g p))\right| p^{\Lambda_{s}}$ is bounded for $p \in P$ and $g$ in a fixed compact set.

Then the series

$$
E_{\rho, f, f^{\prime}}(g)=\sum_{\gamma \in \Gamma / \Gamma_{\infty}}\left(f \otimes\left(f^{\prime} \circ \rho\right)\right)(g \gamma)
$$

converges absolutely and uniformly on any compact set of $G$.

Proof. Since $G=K P$, we have

$$
\begin{aligned}
\left(f \otimes\left(f^{\prime} \circ \rho\right)\right)(g) \cdot a(g)^{\Lambda_{s}} & =\left(f \otimes\left(f^{\prime} \circ \rho\right)\right)(k p) \cdot a(k p)^{\Lambda_{s}} \\
& =\left(f \otimes\left(f^{\prime} \circ \rho\right)\right)(k p) \cdot a(p)^{\Lambda_{s}} \\
& =\left(f \otimes\left(f^{\prime} \circ \rho\right)\right)(k p) \cdot p^{\Lambda_{s}}
\end{aligned}
$$

for $g=k p$ with $k \in K$ and $p \in P$. Hence by (v) $\left|\left(f \otimes\left(f^{\prime} \circ \rho\right)\right)(g) \cdot a(g)^{\Lambda_{s}}\right|$ is bounded for $g \in G$. Therefore, the series defining $E_{f, f^{\prime}, \rho}$ is majorized by a constant times the series

$$
\sum_{\gamma \in \Gamma / \Gamma_{\infty}} a(g \gamma)^{-\Lambda_{s}},
$$

which converges uniformly on any compact set by Lemma 4.1 . Hence the theorem follows. 
Definition 4.3. The series

$$
E_{\rho, f, f^{\prime}}(g)=\sum_{\gamma \in \Gamma / \Gamma_{\infty}}\left(f \otimes\left(f^{\prime} \circ \rho\right)\right)(g \gamma)
$$

is called an Eisenstein series for $\Gamma$ associated to $\rho, f$ and $f^{\prime}$.

\section{Eisenstein series as mixed automorphic forms}

In this section we discuss a special class of Eisenstein series and show that they are mixed automorphic forms. Let $G, P, M, A$, and $U$ be as in $\S 4$. Thus we have decompositions

$$
P=M A U, \quad G=K P=K M A U .
$$

Let $\sigma: K \rightarrow G L(V)$ be a representation of $K$ in a finite-dimensional complex vector space $V$. Let $\pi: M U \rightarrow M$ be the natural projection, and let $K_{M}=\pi(K \cap M U)$. Then $K_{M}$ is a maximal compact subgroup of $M$, and $\sigma$ induces the representation $\sigma_{M}$ of $K_{M}$ given by

$$
\sigma_{M}(\pi(k))=\sigma(k)
$$

for all $k \in K \cap M U$. Let $\Gamma$ be an arithmetic subgroup of $G$, and let $\Gamma_{M}=\pi(\Gamma \cap M U)$ be the corresponding arithmetic subgroup of $M$. We denote by $L^{2}\left(M / \Gamma_{M}, \sigma_{M}\right)$ the space of square-integrable functions $\varphi: M \rightarrow V$ satisfying

$$
\varphi(k m \gamma)=\sigma_{M}(k) \varphi(m)
$$

for all $k \in K_{M}, m \in M$ and $\gamma \in \Gamma_{M}$. Any function $\varphi: M \rightarrow V$ satisfying $\varphi(k m)=\sigma_{M}(k) \varphi(m)$ for $k \in K_{M}$ and $m \in M$ can be extended to a function $\varphi_{G}: G \rightarrow V$ on $G$ by the formula

$$
\varphi_{G}(k m a u)=\sigma(k) \varphi(m)
$$

for all $k \in K, m \in M, a \in A$ and $u \in U$. Then $\varphi_{G}$ is $\sigma$-equivariant; i.e.,

$$
\varphi_{G}(k g)=\sigma(k) \varphi_{G}(g)
$$

for $k \in K$ and $g \in G$. Although a decomposition $g=k m a u$ is not unique, the extension $\varphi_{G}$ is uniquely determined. We shall identify a $\sigma_{M}$-equivariant function $\varphi$ on $M$ and the corresponding $\sigma$-equivariant function $\varphi_{G}$ on $G$. Thus each element of $L^{2}\left(M / \Gamma_{M}, \sigma_{M}\right)$ will be regarded as a function of $G$ into $V$. let

Let $\mathfrak{a}_{\mathbb{C}}^{*}$ be the dual space of the complexification $\mathfrak{a}_{\mathbb{C}}$ of the Lie algebra $\mathfrak{a}$ of $A$, and

$$
\left(\mathfrak{a}_{\mathbb{C}}^{*}\right)^{-}=\left\{\Lambda \in \mathfrak{a}_{\mathbb{C}}^{*} \mid\langle\operatorname{Re} \Lambda+\rho, \alpha\rangle<0 \text { for all } \alpha \in \Sigma^{0}\right\}
$$


where $\Sigma^{0}$ is the set of simple roots of the Lie algebra $g$ of $G$. For $\varphi \in L^{2}\left(M / \Gamma_{M}, \sigma_{M}\right)$ and $\Lambda \in \mathfrak{a}_{\mathbb{C}}^{*}$, we set

$$
\varphi_{\Lambda}(x)=\varphi(x) e^{(\Lambda-\rho)(H(x))}
$$

for $x \in G$, where $H(x)$ denotes $\log a(x)$. Then we have

$$
\varphi_{\Lambda}(k g \gamma)=\sigma(k) \varphi_{\Lambda}(g)
$$

for all $g \in G, k \in K$ and $\gamma \in U(\Gamma \cap P)$. Given $\varphi \in L^{2}\left(M / \Gamma_{M}, \sigma_{M}\right)$ and $\Lambda \in\left(\mathfrak{a}_{\mathbb{C}}^{*}\right)^{-}$, the series

$$
E(\Lambda, \varphi, x)=\sum_{\Gamma / \Gamma \cap P} \varphi_{\Lambda}(x \gamma)
$$

is called an Eisenstein series (see [9]; also see [11], [12], [13], [14], [19]).

Let $\chi$ be a representation of $Z(\mathfrak{g})$ in $V$. We denote by $L^{2}\left(M / \Gamma_{M}, \sigma_{M}, \chi\right)$ the subgroup of $L^{2}\left(M / \Gamma_{M}, \sigma_{M}\right)$ consisting of functions $f: G \rightarrow V$ satisfying the condition

$$
(Y \cdot f)(g)=f(g) \chi(Y)
$$

for all $Y \in Z(\mathfrak{g})$.

LEMMA 5.1. If $\varphi \in L^{2}\left(M / \Gamma_{M}, \sigma_{M}, \chi\right)$ and $\gamma \in U(\Gamma \cap P)$, then there are a positive real number $C$ and a positive integer $N$ such that

$$
\sum_{\Gamma / \Gamma \cap P}\left|\varphi_{\Lambda}(x \gamma)\right| \leq C\|x\|^{N}
$$

for all $x \in G$.

Proof. This follows from [9, Lemma 24].

Let $G^{\prime}$ be another semisimple Lie group, and consider the corresponding objects $K^{\prime}, P^{\prime}, M^{\prime}, A^{\prime}, U^{\prime}, \mathfrak{a}^{\prime}, \mathfrak{a}_{\mathbb{C}}^{\prime *},\left(\mathfrak{a}_{\mathbb{C}}^{\prime *}\right)^{-}$and the representation $\sigma^{\prime}: K^{\prime} \rightarrow G L\left(V^{\prime}\right)$ of $K^{\prime}$ in a finite-dimensional complex vector space $V^{\prime}$. Let $\rho: G \rightarrow G^{\prime}$ be a Lie group homomorphism such that

$$
\rho(K) \subset K^{\prime}, \quad \rho(P) \subset P^{\prime}, \quad \rho(A) \subset A^{\prime} .
$$

As in the case of $G$, for $\varphi^{\prime} \in L^{2}\left(M^{\prime} / \Gamma_{M^{\prime}}^{\prime}, \sigma_{M^{\prime}}^{\prime}\right)$ and $\Lambda^{\prime} \in\left(\mathfrak{a}_{\mathbb{C}}^{\prime *}\right)^{-}$, we set

$$
\varphi_{\Lambda^{\prime}}^{\prime}(y)=\varphi^{\prime}(y) e^{\left(\Lambda^{\prime}-\rho^{\prime}\right)\left(H^{\prime}(y)\right)}
$$

for $y \in G^{\prime}$, where $H^{\prime}(y)$ denotes $\log a^{\prime}(y)$. 
PROPOSITION 5.2. Let $\chi$ and $\chi^{\prime}$ be representations of $Z(\mathfrak{g})$ and $Z\left(\mathfrak{g}^{\prime}\right)$ in $V$ and $V^{\prime}$ respectively, and let $\varphi_{\Lambda} \in L^{2}\left(M / \Gamma_{M}, \sigma_{M}, \chi\right)$ and $\varphi_{\Lambda^{\prime}}^{\prime} \in L^{2}\left(M^{\prime} / \Gamma_{M^{\prime}}^{\prime}, \sigma_{M^{\prime}}^{\prime}, \chi^{\prime}\right)$ with $\Lambda \in\left(\mathfrak{a}_{\mathbb{C}}^{*}\right)^{-}$and $\Lambda^{\prime} \in\left(\mathfrak{a}_{\mathbb{C}}^{\prime *}\right)^{-}$. Then there are a positive real number $C_{0}$ and a positive integer $N_{0}$ such that

$$
\sum_{\gamma \in \Gamma / \Gamma \cap P}\left|\left(\varphi_{\Lambda} \otimes\left(\varphi_{\Lambda^{\prime}}^{\prime} \circ \rho\right)\right)(x \gamma)\right| \leq C_{0}\|x\|^{N_{0}}
$$

for all $x \in G$.

Proof. By Lemma 5.1 there are $C, C^{\prime}, N$, and $N^{\prime}$ such that

$$
\sum_{\gamma \in \Gamma / \Gamma \cap P}\left|\varphi_{\Lambda}(x \gamma)\right| \leq C\|x\|^{N}, \quad \sum_{\gamma^{\prime} \in \Gamma^{\prime} / \Gamma^{\prime} \cap P^{\prime}}\left|\varphi_{\Lambda^{\prime}}^{\prime}\left(x^{\prime} \gamma^{\prime}\right)\right| \leq C^{\prime}\left\|x^{\prime}\right\|^{N^{\prime}}
$$

for all $x \in G$ and $x^{\prime} \in G^{\prime}$. In particular, we have

$$
\begin{aligned}
\sum_{\gamma \in \Gamma / \Gamma \cap P}\left|\varphi_{\Lambda^{\prime}}^{\prime}(\rho(x \gamma))\right| & =\sum_{\gamma \in \Gamma / \Gamma \cap P}\left|\varphi_{\Lambda^{\prime}}^{\prime}(\rho(x) \rho(\gamma))\right| \\
& \leq C^{\prime}\|\rho(x)\|^{N^{\prime}} \leq C^{\prime}\|\rho\|^{N^{\prime}}\|x\|^{N^{\prime}}
\end{aligned}
$$

for all $x \in G$, since $\rho(\gamma) \in \Gamma^{\prime} / \Gamma^{\prime} \cap P^{\prime}$ whenever $\gamma \in \Gamma / \Gamma \cap P$. Thus we obtain

$$
\begin{aligned}
\sum_{\gamma \in \Gamma / \Gamma \cap P}\left|\left(\varphi_{\Lambda} \otimes\left(\varphi_{\Lambda^{\prime}}^{\prime} \circ \rho\right)\right)(x \gamma)\right| & =\sum_{\gamma \in \Gamma / \Gamma \cap P}\left|\varphi_{\Lambda}(x \gamma)\right| \cdot\left|\varphi_{\Lambda^{\prime}}^{\prime}(\rho(x \gamma))\right| \\
& \leq \sum_{\gamma \in \Gamma / \Gamma \cap P}\left|\varphi_{\Lambda}(x \gamma)\right| \cdot \sum_{\gamma \in \Gamma / \Gamma \cap P}\left|\varphi_{\Lambda^{\prime}}^{\prime}(\rho(x \gamma))\right| \\
& \leq C \cdot C^{\prime} \cdot\|\rho\|^{N^{\prime}} \cdot\|x\|^{N+N^{\prime}} ;
\end{aligned}
$$

hence the proposition follows.

We set

$$
E_{\rho, \varphi, \varphi^{\prime}}\left(\Lambda, \Lambda^{\prime}, x\right)=\sum_{\gamma \in \Gamma / \Gamma \cap P}\left(\varphi_{\Lambda} \otimes\left(\varphi_{\Lambda^{\prime}}^{\prime} \circ \rho\right)\right)(x \gamma)
$$

for $x \in G$, which is an Eisenstein series for $\Gamma$ associated to $\rho, \varphi_{\Lambda}$, and $\varphi_{\Lambda^{\prime}}^{\prime}$ in the sense of Definition 4.3 with $\Gamma_{\infty}=\Gamma \cap M U=\Gamma \cap P$ (see $[9, \mathrm{p} .6]$ ).

THEOREM 5.3. The Eisenstein series $E_{\rho, \varphi, \varphi^{\prime}}\left(\Lambda, \Lambda^{\prime}, x\right)$ is a mixed automorphic form for $\Gamma$ of type $\left(\rho, \sigma, \sigma^{\prime}\right)$.

Proof. Recall that $\varphi_{\Lambda}$ can be regarded as a function $\varphi_{\Lambda}: G \rightarrow V$ on $G$ satisfying $\varphi_{\Lambda}(k m a u)=\sigma(k) \varphi_{\Lambda}(m)$. Thus we have

$$
\varphi_{\Lambda}(k g)=\sigma(k) \varphi_{\Lambda}(g)
$$


for all $k \in K$ and $g \in G$. Similarly, we have

$$
\varphi_{\Lambda^{\prime}}^{\prime}\left(k^{\prime} g^{\prime}\right)=\sigma^{\prime}\left(k^{\prime}\right) \varphi_{\Lambda^{\prime}}^{\prime}\left(g^{\prime}\right)
$$

for all $k^{\prime} \in K^{\prime}$ and $g^{\prime} \in G^{\prime}$. Hence it follows that

$$
\left(\varphi_{\Lambda} \otimes\left(\varphi_{\Lambda^{\prime}}^{\prime} \circ \rho\right)\right)(k g)=\left(\sigma \otimes\left(\left.\sigma^{\prime} \circ \rho\right|_{K}\right)\right)(k)\left(\varphi_{\Lambda} \otimes\left(\varphi_{\Lambda^{\prime}}^{\prime} \circ \rho\right)\right)(g)
$$

and

$$
E_{\rho, \varphi, \varphi^{\prime}}(k g)=\left(\sigma \otimes\left(\left.\sigma^{\prime} \circ \rho\right|_{K}\right)\right)(k) E_{\rho, \varphi, \varphi^{\prime}}(g)
$$

for all $k \in K$ and $g \in G$. By Theorem 5.2 we have

$$
\left|\sum_{\gamma \in \Gamma / \Gamma \cap P}\left(\varphi_{\Lambda} \otimes\left(\varphi_{\Lambda^{\prime}}^{\prime} \circ \rho\right)\right)(x \gamma)\right| \leq \sum_{\gamma \in \Gamma / \Gamma \cap P}\left|\left(\varphi_{\Lambda} \otimes\left(\varphi_{\Lambda^{\prime}}^{\prime} \circ \rho\right)\right)(x \gamma)\right| \leq C_{0}\|x\|^{N_{0}},
$$

and consequently the mapping $g \mapsto E_{\rho, \varphi, \varphi^{\prime}}\left(\Lambda, \Lambda^{\prime}, x\right)$ is slowly increasing. Now from the conditions

$$
\left(Y \cdot \varphi_{\Lambda}\right)(g)=\varphi_{\Lambda}(g) \chi(Y), \quad\left(Y^{\prime} \cdot \varphi_{\Lambda^{\prime}}^{\prime}\right)\left(g^{\prime}\right)=\varphi_{\Lambda^{\prime}}^{\prime}\left(g^{\prime}\right) \chi^{\prime}\left(Y^{\prime}\right)
$$

for $Y \in Z(\mathfrak{g}), Y^{\prime} \in Z\left(\mathfrak{g}^{\prime}\right), g \in G$ and $g^{\prime} \in G^{\prime}$, it follows that $\varphi_{\Lambda}$ is $Z(\mathfrak{g})$-finite and $\varphi_{\Lambda^{\prime}}^{\prime}$ is $Z\left(\mathfrak{g}^{\prime}\right)$-finite; hence, as in the proof of Proposition 2.3, $\varphi_{\Lambda} \otimes\left(\varphi_{\Lambda^{\prime}}^{\prime} \circ \rho\right)$ is $Z(\mathfrak{g})$-finite. Therefore $E_{\rho, \varphi, \varphi^{\prime}}$ is also $Z(\mathfrak{g})$-finite, and the theorem follows.

\section{REFERENCES}

1. W. Baily, Introductory lectures on automorphic forms, Princeton Univ. Press, Princeton, N.J., 1973.

2. W. Baily and A. Borel, Compactification of arithmetic quotients of bounded symmetric domains, Ann. Math 84 (1966), 442-528.

3. A. Borel, Introduction to automorphic forms, Proc. Symp. Pure Math., vol. 9, Amer. Math. Soc., Providence, R.I., 1966, pp. 199-210.

4. A. Borel and H. Jacquet, Automorphic forms and automorphic representations, Proc. Symp. Pure Math., vol. 33-I, Amer. Math. Soc., Providence, R.I., 1979, pp. 189-202.

5. S. Gelbart, Automorphic forms on adele groups, Princeton Univ. Press, Princeton, N.J., 1975.

6. S. Gelbart and F. Shahidi, Analytic properties of automorphic L-functions, Academic Press New York, 1988.

7. R. Godement, Introduction à la théorie de Langlands, Sém. Bourbaki 19 (1966/67), Exposé 321.

8. Harish-Chandra, Representations of semi-simple Lie groups: VI, Amer. J. Math. 78 (1956), 564-628.

9. Harish-Chandra, Automorphic forms on semisimple Lie groups, Springer-Verlag, Heidelberg, 1968.

10. B. Hunt and W. Meyer, Mixed automorphic forms and invariants of elliptic surfaces, Math. Ann. 271 $1985,53-80$.

11. T. Kubota, Elementary theory of Eisenstein series, John Wiley, New York, 1973.

12. R. Langlands, Eisenstein series, Proc. Symp. Pure Math., vol. 9, Amer. Math. Soc., Providence, R.I., 1966, pp. 235-252.

13. R. Langlands, Euler products, Yale University Press, 1967.

14. R. Langlands, On the functional equations satisfied by Eisenstein series, Springer-Verlag, Heidelberg, 1977. 
15. M. H. Lee, Mixed cusp forms and holomorphic forms on elliptic varieties, Pacific J. Math. 132 (1988), 363-370.

16. M. H. Lee, Mixed cusp forms and Poincaré series, Rocky Mountain J. Math. 23 (1993), 1009-1022.

17. M. H. Lee, Mixed Siegel modular forms and Kuga fiber varieties, Illinois J. Math. 38 (1994), 692-700.

18. M. H. Lee, Mixed automorphic vector bundles on Shimura varieties, Pacific J. Math. 173 (1996), 105-126.

19. M. Osborne and G. Warner, The theory of Eisenstein systems, Academic Press, New York, 1990.

20. I. Satake, Algebraic structures of symmetric domains, Princeton Univ. Press, Princeton, N.J., 1980.

21. A. Selberg, Harmonic analysis and discontinuous groups in weakly symmetric Riemannian spaces with applications to Discrete series, J. Indian Math. 20 (1956), 47-87.

\title{
UNIVERSITY OF NORTHERN IOWA
}

\author{
CEDAR FALLS, IOWA
}

